\title{
Kondo signature in heat transfer via a local two-state system
}

\author{
Keiji Saito ${ }^{1}$ and Takeo Kato ${ }^{2}$ \\ ${ }^{1}$ Department of Physics, Keio University, Yokohama 223-8522, Japan \\ ${ }^{2}$ Institute for Solid State Physics, the University of Tokyo, Kashiwa, Chiba 277-8581, Japan
}

(Dated: July 13, 2018)

\begin{abstract}
We study the Kondo effect in heat transport via a local two-state system. This system is described by the spin-boson Hamiltonian with Ohmic dissipation, which can be mapped onto the Kondo model with anisotropic exchange coupling. We calculate thermal conductance by the Monte Carlo method based on the exact formula. Thermal conductance has a scaling form $\kappa=\left(k_{B}^{2} T_{K} / \hbar\right) f\left(\alpha, T / T_{K}\right)$, where $T_{K}$ and $\alpha$ indicate the Kondo temperature and dimensionless coupling strength, respectively. Temperature dependence of conductance is classified by the Kondo temperature as $\kappa \propto\left(T / T_{K}\right)^{3}$ for $T \ll T_{K}$ and $\kappa \propto\left(k_{B} T / \hbar \omega_{c}\right)^{2 \alpha-1}$ for $T \gg T_{K}$. Similarities to the Kondo signature in electric transport are discussed.
\end{abstract}

PACS numbers: 44.10.+i,72.10.Fk,05.60.Gg

Heat and electric transport are ubiquitous phenomena. Both phenomena have several similarities as well as dissimilarities. Fourier's law in heat transport corresponds to Ohm's law in electric transport, and these laws are commonly categorized as diffusive transport. We also note that heat transport shows unique anomaly in low dimensions $[1-3]$. Ballistic transport leads to the quantization of conductance in electric [4] as well as heat transport [5]. The conductance quantum was measured in mesoscopic electric conduction in 1988 [6], and much later, the version of heat transport was also measured [7]. Recently, the concept of thermal diode has also been discussed [8], and an experiment has been conducted for demonstrating this [9]. Recent progress in transport studies strongly indicates that heat transport analogue exists for many categories of electric transport.

In this paper, we consider heat transfer between phononic reservoirs via a local two-state system, and aim to clarify the signature of the Kondo effect in heat transport. This setup is analogous to electric transport via quantum dots, which is a typical and simplest example of quantum transport through a zero-dimensional physical object. In quantum-dot systems, the Kondo effect is an interesting and famous phenomenon induced by electron correlation [4, 10, 11]. The Coulomb blockade for electron tunneling is overcome by the formation of the Kondo singlet between a localized electric spin and conduction electrons. Because of this effect, electric conductance is nontrivially enhanced and can eventually reach conductance quantum.

We consider heat transfer via a two-level system modeled by the spin-boson system with Ohmic dissipation [12, 13]. In the equilibrium situation, low-energy physics of this model is understood in terms of Kondo physics through mapping onto the anisotropic Kondo model [12 14]. Let $\alpha$ be a dimensionless coupling strength between the spin and bosonic reservoirs in the spin-boson Hamiltonian (exact definition is given below in Eq. (2)). The weak coupling region of $\alpha<1$ is

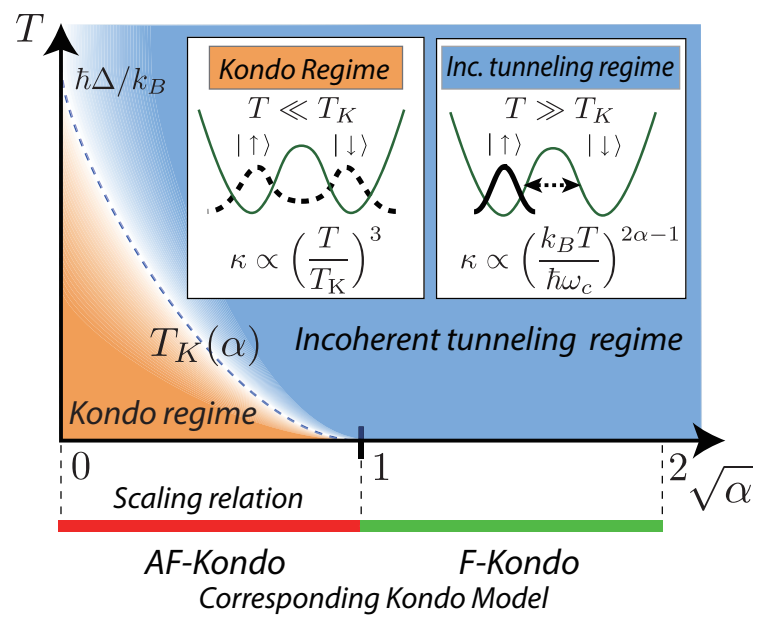

FIG. 1: (color online) Schematic summary of results. The Kondo regime and the incoherent tunneling regime are shown in the space of temperature versus dimensionless coupling strength $\alpha$. The dashed line indicates the Kondo temperature $T_{K}$, defined in Eq. (3). Mapping onto the corresponding parameter region of the Kondo model is shown below the figure; the region of $0 \leq \alpha<1$ corresponds to the Kondo model with antiferromagnetic exchange coupling (AF-Kondo), and $1<\alpha \leq 4$ to the one with ferromagnetic exchange coupling (F-Kondo). The inset shows schematic quantum states as well as main findings on thermal conductance (Eqs. (10)-(11)). The scaling relation Eq. (9) holds for $0 \leq \alpha<1$.

mapped onto the antiferromagnetic parameter regime in the Kondo model, where the Kondo singlet is formed between a quantum dot and conduction electrons at sufficiently low temperatures. On the other hand, the region of $\alpha>1$ is mapped onto the ferromagnetic parameter regime, in which only a trivial spin-doublet state is realized [15]. At zero temperature, quantum states in the spin-boson model are clearly separated by quantum phase transition of the Kosterlitz-Thouless type at $\alpha=1$, where the local two-state forms one bonding state for $\alpha<1$ and two degenerate states for $\alpha>1$. The Kondo 
temperature, a unique temperature scale characteristic of the Kondo effect, is defined as a function of $\alpha$, and in the regime below the Kondo temperature (referred to as the Kondo regime), the local two-state is strongly and coherently correlated with the phononic environment. See Fig. 1 1 for schematic explanation.

The spin-boson model is a minimal model that describes molecular junctions [16], a superconducting circuit 12], and a photonic waveguide with a local two-level system [17], etc.; heat transport has also been studied intensively [18 21]. These include thermal rectification effects [19], the cotunneling process [20], and fluctuations in current [21]. From the underlying Kondo physics in the equilibrium situation, it is also of general interest to analyze low-temperature properties in heat transport. We note that the cotunneling mechanism has been studied [20]. However, so far, no systematic studies have yet been conducted to get the Kondo signature induced by higher-order processes beyond cotunneling. This is the first study that shows the universal aspects arising from the underlying Kondo physics based on the exact formula of heat current. We indicate similarities and dissimilarities of the Kondo signature between heat and electric transport via a zero-dimensional system.

Model and exact current formula. - The local two-level system attached to two phononic reservoirs is described by the following spin-Boson Hamiltonian:

$H=\frac{\hbar \Delta}{2} \sigma_{x}+\sum_{\nu=L, R} \sum_{k} \frac{\hbar \sigma_{z}}{2} \lambda_{\nu k}\left(b_{\nu k}+b_{\nu k}^{\dagger}\right)+\hbar \omega_{\nu k} b_{\nu k}^{\dagger} b_{\nu k}$,

where $\Delta$ is the tunneling frequency between the two states, i.e., the up-spin state $|\uparrow\rangle$ and the down-spin state $|\downarrow\rangle$. The operator $\sigma_{\mu}(\mu=x, y, z)$ is the Pauli matrix, and $b_{\nu k}$ is the annihilation operator of phonons with the wavenumber $k$ in the $\nu$ th reservoir. The two reservoirs are characterized by the spectral density defined as $I_{\nu}(\omega)=\sum_{k} \lambda_{\nu k}^{2} \delta\left(\omega-\omega_{\nu k}\right)$. We assume the Ohmic dissipation for both reservoirs as follows:

$$
\begin{aligned}
& I_{\nu}(\omega)=2 \alpha_{\nu} \tilde{I}(\omega), \quad \alpha=\alpha_{L}+\alpha_{R}, \\
& \tilde{I}(\omega)=\omega \theta\left(\omega_{c}-\omega\right) \theta(\omega),
\end{aligned}
$$

where $\alpha_{\nu}$ is the dimensionless coupling strength between the system and the $\nu$ th reservoir. The cutoff energy $\hbar \omega_{c}$ is assumed to be sufficiently large compared to the system's energy scale. The Hamiltonian (1) can be mapped onto the Kondo Hamiltonian with anisotropic exchange coupling [14]. The in-plane and out-of-plane exchange parameters $J_{\|}$and $J_{\perp}$ are related to the parameters in the spin-Boson model as $\alpha=\left[1-(2 / \pi) \arctan \left(\pi \rho_{0} J_{\|} / 4\right)\right]^{2}$ and $\Delta=\rho_{0} \omega_{c} J_{\perp}$ respectively, where $\rho_{0}$ is a density of state (See the supplementary material 15]). The Kondo temperature $T_{K}$ is defined by renormalization group analysis [12]:

$$
T_{K}=\left\{\begin{array}{lll}
\left(\hbar / k_{B}\right) g_{\alpha} \Delta\left(\Delta / \omega_{c}\right)^{\alpha /(1-\alpha)} & \cdots & 0 \leq \alpha<1, \\
0 & \cdots & \alpha \geq 1,
\end{array}\right.
$$

where $k_{B}$ is the Boltzmann constant. The factor $g_{\alpha}$ is a nonuniversal constant, and throughout the paper, we take $g_{\alpha}=[\Gamma(1-2 \alpha) \cos (\pi \alpha)]^{(1 / 2(1-\alpha))}$ with the Gamma function $\Gamma(x)[13]$.

The exact formula of heat current is derived with the standard procedure of the Keldysh formalism. The initial density matrix is prepared as the product form of equilibrium states of the system and left and right reservoirs. The temperatures of the left and right reservoirs are $T_{L}$ and $T_{R}$, respectively. The heat current operator from the $\nu$ th reservoir into the system is given by

$$
J_{\nu}=i \frac{\sigma_{z}}{2} \sum_{k} \lambda_{\nu k} \hbar \omega_{\nu k}\left(-b_{\nu k}+b_{\nu k}^{\dagger}\right) .
$$

Expressing this with the Keldysh green's function [18, 22], and noting the Gaussian properties for bosonic variables, we derive a formal expression for current [23]: $\left\langle J_{\nu}\right\rangle=\hbar^{2} / 2 \int_{0}^{\infty} d \omega \omega\left[\chi^{\prime \prime}(\omega) I_{\nu}(\omega) n_{\nu}(\omega)-i C(\omega) I_{\nu}(\omega) / 2\right]$ where $C(\omega)$ is the Fourier transform of the lesser green function, $C\left(t, t^{\prime}\right)=-i \hbar^{-1}\left\langle\sigma_{z}\left(t^{\prime}\right) \sigma_{z}(t)\right\rangle$, and $n_{\nu}(\omega)$ is the Bose-Einstein distribution of temperature $T_{\nu}$. The symbol $\langle\cdots\rangle$ implies taking an average at a steady state. The function $\chi^{\prime \prime}(\omega)$ is the imaginary part of the Fourier's transform for the response function of the spin, which is defined as $\chi\left(t, t^{\prime}\right)=i \hbar^{-1} \theta\left(t-t^{\prime}\right)\left\langle\left[\sigma_{z}(t), \sigma_{z}\left(t^{\prime}\right)\right]\right\rangle$. Note that the well-known Landauer-type formula for the ballistic transport cannot be derived because the spin-boson Hamiltonian is not bilinear. However, we can derive an extended version of the Landauer-type formula by considering zero dimensionality of the system and the conservation law of current $\left\langle J_{L}\right\rangle+\left\langle J_{R}\right\rangle=0$ [24, 25]. Then the exact heat-current formula is given by

$$
\left\langle J_{L}\right\rangle=\frac{\hbar^{2} \alpha \gamma}{4} \int_{0}^{\infty} d \omega \omega \chi^{\prime \prime}(\omega) \tilde{I}(\omega)\left[n_{L}(\omega)-n_{R}(\omega)\right],
$$

where $\gamma=4 \alpha_{L} \alpha_{R} / \alpha^{2}$ is an asymmetry factor. In the linear response regime, thermal conductance defined by $\kappa=d\left\langle J_{L}\right\rangle /\left.d T_{L}\right|_{T_{L} \rightarrow T_{R}=T}$ is given by

$$
\kappa=\frac{k_{B} \hbar \alpha \gamma}{4} \int_{0}^{\omega_{c}} d \omega S_{\alpha}(\omega) \omega^{2}\left[\frac{\beta \hbar \omega / 2}{\sinh (\beta \hbar \omega / 2)}\right]^{2},
$$

where we substitute the Ohmic spectral density for $\tilde{I}(\omega)$, and $S_{\alpha}(\omega)$ is the spectral function defined as

$$
S_{\alpha}(\omega)=\chi^{\prime \prime}(\omega) / \omega .
$$

The formulas (5) and (6) are the basis of our calculation. Before discussing the Kondo signature in heat transport, it is helpful to examine the current formula derived by the quantum master equation approach by Segal and Nitzan [19]. By utilizing their expression of current, thermal conductance is obtained as

$$
\kappa_{W C}=\frac{k_{B} \alpha \gamma}{4} \frac{\pi \Delta}{2 n(\Delta)+1}\left[\frac{\beta \hbar \Delta / 2}{\sinh (\beta \hbar \Delta / 2)}\right]^{2},
$$




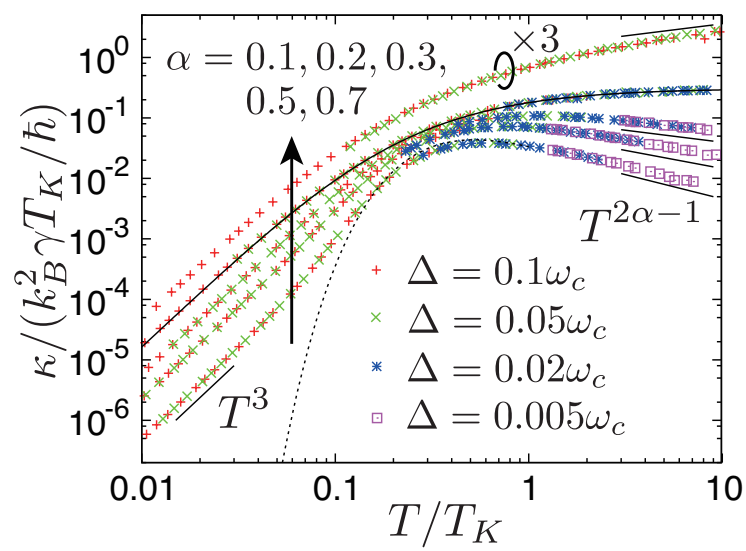

FIG. 2: (color online) Low-temperature behavior of the conductance calculated with the Monte Carlo simulation. The data represent results for $\alpha=0.1,0.2,0.3,0.5$, and 0.7 from bottom to top. The solid line is an exact result for the Toulouse point $\alpha=1 / 2$. The figure clearly shows scaling form $\kappa(T) \sim\left(k_{B}^{2} \gamma T_{K} / \hbar\right) f\left(\alpha, T / T_{K}\right)$ and the $T^{3}$-law in the low-temperature regime. The dotted line indicates $\kappa_{W C}$ for $\alpha=0.1$. For $T \gg T_{K}, \kappa$ depends on $T^{2 \alpha-1}$.

where $n(\omega)$ denotes the Bose-Einstein distribution of temperature $T$. This expression is reproduced from the exact formula (6) in the weak coupling limit $(\alpha \rightarrow 0)$. This is checked by substituting the zeroth order of the spectral function, namely the expression for the isolated system: $S_{0}(\omega)=\pi \delta(\omega-\Delta) /[\hbar \omega(2 n(\omega)+1)]$.

We note that at low temperatures $k_{B} T \ll \hbar \Delta$, the weak coupling approximation predicts the Schottky-type temperature dependence as $\kappa_{W C} \propto e^{-\hbar \Delta / k_{B} T}$, leading to the exponential suppression of heat current. This property is analogous to the Coulomb-blockade phenomenon in electric conduction, where electric conductance is exponentially suppressed at low temperatures because of the excess electrostatic energy of electrons in a quantum dot. However, as shown below, finite coupling to reservoirs remarkably changes the transport properties showing nontrivial universal properties.

Numerical method. - In the subsequent sections, we focus on thermal conductance. In the exact formula (6), the spectral function $S_{\alpha}(\omega)$ includes the entire information on the many-body effect. We calculate $S_{\alpha}(\omega)$ by the Monte Carlo method, as follows. (i) We note that the path-integral representation of the equilibrium partition function for the Hamiltonian (1) is mapped onto a one-dimensional Ising model with long-range interaction (for details, see the supplementary material [15]). (ii) For this Ising model, the Monte Carlo simulation with the Wolff algorithm [26] is performed to obtain the spin-spin correlation, which is equivalent to Matsubara's green function $\mathcal{G}(u)=\left\langle e^{u H} \sigma_{z} e^{-u H} \sigma_{z}\right\rangle_{\text {eq }}$, where $\langle\cdots\rangle_{\text {eq }}$ implies an equilibrium average. (iii) From the Fourier transformation of Matsubara's green function $\mathcal{G}(i \xi)$, we calculate $\chi(\omega)$ using numerical analytic continuation $\chi(\omega)=\mathcal{G}(i \xi \rightarrow \omega+i \delta)$ by the Padé approximation [27, 28].

Kondo signature in thermal conductance. - In Fig. 2 , we show temperature dependence of the thermal conductance calculated with the abovementioned numerical procedure. The data indicate results for $\alpha<1$, which corresponds to the antiferromagnetic coupling regime $\left(J_{\|}<0\right)$ in the mapped Kondo model. The horizontal axis indicates the temperature scaled by the Kondo temperature $T_{K}$, and the vertical axis indicates the conductance scaled by $k_{B}^{2} \gamma T_{K} / \hbar$. To confirm numerical accuracy, we note that $\alpha=1 / 2$ is an exactly soluble point, called the Toulouse point, where the spectral function $S_{1 / 2}$ is given by 13 ]

$$
S_{1 / 2}(\omega)=\operatorname{Im}\left[\frac{4}{\hbar \pi \omega^{2}} \frac{k_{B} T_{K}}{\hbar \omega+i\left(k_{B} T_{K} / \hbar\right)}\left[\psi\left(z^{\prime}\right)-\psi\left(z^{\prime \prime}\right)\right]\right],
$$

where $\psi(x)$ is the digamma function with the variables $z^{\prime}=1 / 2+\beta k_{B} T_{K} /(4 \pi)$ and $z^{\prime \prime}=1 / 2+\beta k_{B} T_{K} /(4 \pi)-$ $i \beta \hbar \omega /(2 \pi)$. In Fig. 2 we also show the exact values for the Toulouse point by a solid line. Our numerical results clearly agree with the exact results. Another evidence of accuracy is given by a validation of the Shiba relation [29] for arbitrary coupling strength [15].

Fig. 2 shows that all the data collapse onto one curve for each value of $\alpha$ regardless of the tunneling frequency $\Delta$. This implies emergence of the scaling form in $\kappa$ :

$$
\kappa(T)=\left(k_{B}^{2} \gamma T_{K} / \hbar\right) f\left(\alpha, T / T_{K}\right) .
$$

This scaling form is an indication of the Kondo effect in heat transfer. In addition, conductance is proportional to $T^{3}$ at sufficiently low temperatures $T \ll T_{K}$. At high temperatures $T \gg T_{K}$, conductance depends on temperature as $T^{2 \alpha-1}$, which intriguingly implies that the coupling strength determines monotonicity of temperature dependence. These numerical findings are the first main results of this study.

For comparison, $\kappa_{W C}$ given by Eq. (8) is shown for $\alpha=0.1$ in Fig. 2 by a dotted line. Although $\kappa_{W C}$ is quantitatively good around the Kondo temperature, it deviates from the numerical results at lower temperatures, showing exponential reduction. Enhanced heat transport from $\kappa_{W C}$ is analogous to enhanced electronic transport via quantum dots in the Kondo regime. However, note that conductance does not reach the universal quantum of thermal conductance, $g(T)=\pi k_{B}^{2} T /(6 \hbar)$, which is linear in $T$. Heat transfer is generally sensitive to the scattering mechanism, and hence conductance tends to be reduced. This aspect is dissimilar to the Kondo signature in electric transport, where electric conductance can reach the conductance quantum.

To explain the universal $T^{3}$-law for $T \ll T_{K}$, we first consider the spectral function at the Toulouse point. The spectral function $S_{1 / 2}(\omega)$ is expanded with respect to temperature and frequency as in the form $S_{1 / 2}(\omega)=$ 


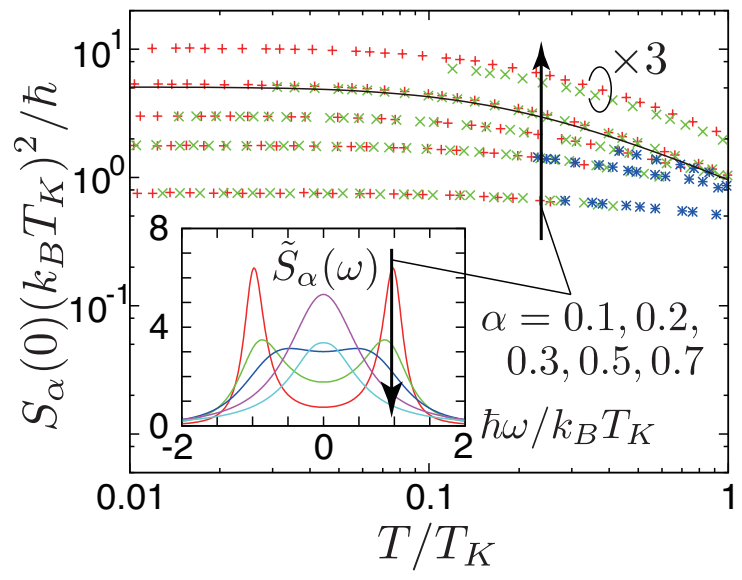

FIG. 3: (color online) Temperature dependence of $S_{\alpha}(0)$ for various $\alpha$ obtained by the Monte Carlo method. Legends are the same as those for Fig. 2 The solid line shows the analytic solution at the Toulouse point $(\alpha=1 / 2)$. The inset shows $\omega$ dependence of the scaled spectral function $\tilde{S}_{\alpha}(\omega)=$ $S_{\alpha}(\omega)\left(k_{B} T_{K}\right)^{2} / \hbar$ for the low-temperature regime.

$\hbar /\left(k_{B} T_{K}\right)^{2}\left[16 / \pi+O\left(\left(\hbar \omega / k_{B} T_{K}\right)^{2}\right)+O\left(\left(T / T_{K}\right)^{2}\right)\right]$, i.e., the second order follows after the zeroth order. In Fig. 3 we show the temperature dependence of $S_{\alpha}(0)$. The inset shows $\omega$ dependence of $S_{\alpha}(\omega)$ for various $\alpha$ in the lowtemperature regime. This figure shows that the spectral function generally has the same type of expansion as for the Toulouse point. Hence, the spectral function is safely approximated as $S_{\alpha}(\omega) \sim \hbar /\left(k_{B} T_{K}\right)^{2} h_{\alpha}$ for a sufficiently low-temperature and small-frequency regime, where $h_{\alpha}$ is a non-universal constant. From this, conductance is approximated as

$$
\begin{aligned}
\kappa & \simeq \frac{\hbar \alpha \gamma}{4 k_{B} T_{K}^{2}} \int_{0}^{\infty} d \omega h_{\alpha} \omega^{2}\left[\frac{\beta \hbar \omega / 2}{\sinh (\beta \hbar \omega / 2)}\right]^{2} \\
& \simeq\left(k_{B}^{2} T_{K} \mathcal{N} / \hbar\right)\left(T / T_{K}\right)^{3} \quad \text { for } T \ll T_{K},
\end{aligned}
$$

where $\mathcal{N}=\pi^{4} h_{\alpha} \alpha \gamma / 15[30]$.

We next consider the higher-temperature regime of $T_{K} \ll T \ll \hbar \omega_{c} / k_{B}$. We start with the fluctuation dissipation theorem: $S_{\alpha}(\omega)=D(\omega) /[\hbar \omega \operatorname{coth}(\beta \hbar \omega / 2)]$, where $D(\omega)$ is the Fourier transform of the symmetrized correlation function $D(t)=\left\langle\left\{\sigma^{z}(t), \sigma^{z}\right\}\right\rangle_{\mathrm{eq}} / 2-\left\langle\sigma^{z}\right\rangle_{\mathrm{eq}}^{2}$. Since the temperature is much higher than the Kondo temperature, the spin dynamics is incoherent and the correlation $D(t)$ decays exponentially. Hence, $S_{\alpha}(\omega)$ can be written as $S_{\alpha}(\omega) \simeq 2 \zeta /\left[\left(\omega^{2}+\zeta^{2}\right) \hbar \omega \operatorname{coth}(\beta \hbar \omega / 2)\right]$ where $\zeta$ is the decay rate of $D(t)$. We now employ the decay rate obtained using the noninteracting blip approximation [12, 31, 32]; $\zeta \propto\left(\Delta^{2} / \omega_{c}\right)\left(\beta \hbar \omega_{c}\right)^{1-2 \alpha}$. By plugging this into the conductance formula (6), we get an approximation for conductance as

$$
\kappa \simeq \mathcal{C} \frac{k_{B} \Delta^{2}}{\omega_{c}}\left(\frac{k_{B} T}{\hbar \omega_{c}}\right)^{2 \alpha-1} \text { for } T_{K} \ll T \ll \hbar \omega_{c} / k_{B}
$$

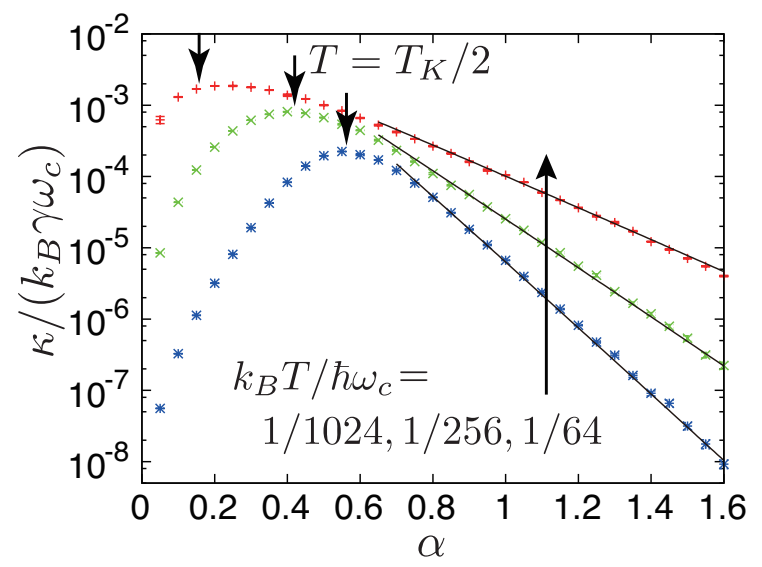

FIG. 4: (color online) Dependence of $\kappa$ on the couping strength $\alpha$ for three temperatures. We set $\Delta=0.05 \omega_{c}$. Conductance is numerically found to be maximum around $\alpha$ satisfying $T=T_{K} / 2$, which is shown by small arrows. The solid line shows the incoherent tunneling approximation (11).

where $\mathcal{C}$ is a prefactor that weakly depends on coupling strength. This explains the numerical observation on temperature dependence in the high-temperature region $T_{K} \ll T$. Note that Eq. (11) holds at arbitrary temperatures for $\alpha>1$ since the Kondo temperature is zero.

We finally discuss coupling-strength dependence. In Fig. 4. conductance is shown as a function of $\alpha$ for fixed temperatures. As $\alpha$ increases, conductance first increases rapidly, and then decreases exponentially. We note that the Kondo temperature is rapidly suppressed as $\alpha$ increases (see Eq. (3) and Fig. 11). The former increase corresponds to the Kondo regime $\left(T \ll T_{K}(\alpha)\right)$, and is due to the suppression of $T_{K}$ (see Eq. (10)). On the other hand, the latter exponential decrease corresponds to the incoherent tunneling regime $\left(T \gg T_{K}(\alpha)\right)$, and is caused by the factor $\left(k_{B} T / \hbar \omega_{c}\right)^{2 \alpha-1}$ in Eq. (11). The exponential reduction of conductance is an evidence of the strong suppression of tunneling due to large dissipation, consistently with an indication in 33, 34]. Clear exponential suppression explained by Eq. (11) is the second main result.

Summary. - We considered thermal transport via a local two-state system, and investigated low-temperature properties on the basis of the exact formula (6). Our findings are schematically summarized in Fig. 1 We obtained, as Kondo-signature, the scaling form (9), and conductance classified by the Kondo temperature; Eq. (10) for $T \ll T_{K}$ and Eq. (11) for $T_{K} \ll T \ll$ $\hbar \omega_{c} / k_{B}$. The exponential reduction of conductance for large coupling is explained by the formula Eq. (11). We hope that our study motivates further research on lowenergy heat transfer via local systems. Effect of bias, other types of dissipation 35 and far-from-equilibrium effect, e.g., for problems in [36, 37] will be intriguing subjects in this direction. 


\section{Acknowledgement}

We are grateful to Mikio Eto, Teemu Ojanen, Rui Sakano and Dvira Segal for useful comments. We also thank Ulrich Weiss for showing the derivation of $g_{\alpha}$. KS was supported by MEXT (23740289). TK was supported by JSPS KAKENHI Grant Number 24540316.

[1] F. Bonetto, J. L. Lebowitz and L. Rey-Bellet, in Mathematical Physics 2000, edited by A. Fokas et. al. (Imperial College Press, London, 2000), p. 128.

[2] S. Lepri, R. Livi and A. Politi, Phys. Rep. 377, 1 (2003).

[3] A. Dhar, Adv. Phys. 57, 457 (2008).

[4] S. Datta, Quantum Transport: Atom to Transistor, (Cambridge, 2005).

[5] L. G. C. Rego and G. Kirczenow, Phys. Rev. Lett. 81, 232 (1998).

[6] B. J. Wees et al., Phys. Rev. Lett. 60, 848 (1988).

[7] K. Schwab et al., Nature (London) 404, 974 (2000); H.-Y. Chiu et al., Phys. Rev. Lett. 95, 226101 (2005).

[8] N. Li, J. Ren, L. Wang, G. Zhang, P. Hänggi and B. Li, Rev. Mod. Phys. 84, 1045 (2012).

[9] C. W. Chang, D. Okawa, A. Majumdar and A. Zettl, Science 314, 1121 (2006)

[10] D. Goldhaber-Gordon et al., Nature 391, 156 (1998); W. van der Wiel et al., Science 289, 2105 (2000).

[11] A. C. Hewson, The Kondo Problem to Heavy Fermions, (Cambridge University Press, Cambridge, 1997).

[12] A. J. Leggett, S. Chakravarty, A. T. Dorsey, M. P. A. Fisher, A. Garg and W. Zwerger, Rev. Mod. Phys. 59, 1 (1987).

[13] U. Weiss, Quantum Dissipative Systems, (World Scientific, Singapore, 1999).

[14] F. Guinea, V. Hakim and A. Muramatsu, Phys. Rev. B 32, 4410 (1985); F. Guinea, Phys. Rev. B 32, 4486 (1985).

[15] In the supplementary material, we present details on mapping onto the anisotropic Kondo model, calculation of heat current from the quantum master equation approach, relation to an Ising model with long-range interaction, the Shiba relation, and the cotunneling formula.
[16] A. Nitzan, Chemical Dynamics in Condensed Phases: Relaxation, Transfer, and Reactions in Condensed Molecular Systems, (Oxford, 2006).

[17] K. Le Hur, Phys. Rev. B 85, 140506 (2012).

[18] K. A. Velizhanin, M. Thoss and H. Wang, J. Chem. Phys. 133084503 (2010).

[19] D. Segal and A. Nitzan, Phys. Rev. Lett. 94, 034301 (2005); J. Chem. Phys. 122, 194704 (2005).

[20] T. Ruokola and T. Ojanen, Phys. Rev. B 83, 045417 (2011).

[21] L. Nicolin and D. Segal, Phys. Rev. B 84, 161414 (2011).

[22] J.-S. Wang, J. Wang and N. Zeng, Phys. Rev. B 74, 033408 (2006).

[23] K. Saito, Europhys Lett. 83, 50006 (2008).

[24] Y. Meir and N. S. Wingreen, Phys. Rev. Lett. 68, 2512 (1992).

[25] T. Ojanen and A. -P. Jauho, Phys. Rev. Lett. 100, 155902 (2008).

[26] U. Wolff, Phys. Rev. Lett. 62, 361 (1989).

[27] K. Völker, Phys. Rev. B 58, 1862 (1998).

[28] C. Brezinski and Z. M. Redivo, Extrapolation Methods. Theory and Practice. (North-Holland, 1991).

[29] H. Shiba, Prog. Theor. Phys. 54, 967 (1975).

[30] The $T^{3}$ law in the Kondo regime differs from a recent result by renormalization analysis based on cotunneling picture $\kappa \propto T^{3-2 \alpha}$ [20]. We note that only cotunneling process also leads to the $T^{3}$ law, but fails in reproducing a correct energy scale. Detailed discussion including correction of renormalization procedure is given in the supplementary material [15].

[31] H. Grabert and U. Weiss, Phys. Rev. Lett. 54, 1605 (1985).

[32] M. P. A. Fisher and A. T. Dorsey, Phys. Rev. Lett. 54, 1609 (1985).

[33] K. A. Velizhanin, H. Wang and M. Thoss, Chem. Phys. Lett. 460, 325 (2008).

[34] L. Nicolin and D. Segal, J. Chem. Phys. 135, 164106 (2011).

[35] T. Kato and K. Saito, in preparation.

[36] D. Segal, arXiv:1303.5815.

[37] J. Ren, P. Hänggi and B. Li, Phys. Rev. Lett. 104, 170601 (2010), T. Chen, X.B. Wang and J. Ren, Phys. Rev. B 87, 144303 (2013).

Supplementary Material for

"Kondo signature in heat transfer via a local two-state system"

Anisotropic Kondo model. - The Hamiltonian of the anisotropic Kondo model is given by

$$
\begin{aligned}
H_{A K} & =\sum_{k} \sum_{\sigma=\uparrow, \downarrow} \epsilon_{k} c_{k \sigma}^{\dagger} c_{k \sigma} \\
& +J_{\perp} \sum_{k, k^{\prime}}\left(c_{k \uparrow}^{\dagger} c_{k^{\prime} \downarrow} S^{-}+c_{k \downarrow}^{\dagger} c_{k^{\prime} \uparrow} S^{+}\right) \\
& +\frac{J_{\|}}{2} \sum_{k, k^{\prime}}\left(c_{k \uparrow}^{\dagger} c_{k^{\prime} \uparrow}-c_{k \downarrow}^{\dagger} c_{k^{\prime} \downarrow}\right) S_{z},
\end{aligned}
$$

where $c_{k \sigma}$ is an annihilation operator of the fermion of the wave number $k$ and spin $\sigma$. Operators $S_{\mu}(\mu=x, y, z)$ is the spin operator by localized electron inside quantumdot and $S^{ \pm}=S_{x} \pm i S_{y}$. The parameters $J_{\|}$and $J_{\perp}$, respectively, represent the in-plane and out-of-plane exchange parameters. When $J_{\|}>0$, the Hamiltonian describes the antiferromagnetic Kondo (AF-Kondo) model, while $J_{\|}<0$ corresponds to the ferromagnetic Kondo (F-Kondo) model. The correspondence between the anisotropic Kondo model and the spin-boson model via 
bosonization [1, 2] implies

$$
\begin{aligned}
\alpha & =\left[1-\frac{2}{\pi} \arctan \left(\pi \rho_{0} J_{\|} / 4\right)\right]^{2}, \\
\Delta & =\rho_{0} \omega_{c} J_{\perp}
\end{aligned}
$$

where $\rho_{0}$ is the density of the state of conduction electrons. These relations indicate that the regime $-\infty<$ $\rho J_{\|}<\infty$ is mapped onto $4>\alpha>0$. The phase transition point $\alpha=1$ in the spin-boson model corresponds to the transition point between the AF-Kondo and F-Kondo regime (see Fig. 1 in the main text).

Exact formula of heat transfer. - The formal current formula is given by

$$
\left\langle J_{\nu}\right\rangle=\frac{\hbar^{2}}{2} \int_{0}^{\infty} d \omega \omega\left[\chi^{\prime \prime}(\omega) I_{\nu}(\omega) n_{\nu}(\omega)-i C(\omega) I_{\nu}(\omega) / 2\right]
$$

We define a quantity

$$
r_{\nu}=\frac{\int_{0}^{\infty} d \omega \hbar \omega C(\omega) I_{\nu}(\omega)}{\sum_{\nu^{\prime}=L, R} \int_{0}^{\infty} d \omega \hbar \omega C(\omega) I_{\nu^{\prime}}(\omega)} .
$$

From the conservation law $\left\langle J_{L}\right\rangle+\left\langle J_{R}\right\rangle=0$, the current formula is rewritten as

$$
\begin{aligned}
\left\langle J_{L}\right\rangle & =r_{R}\left\langle\mathcal{J}_{L}\right\rangle-r_{L}\left\langle\mathcal{J}_{R}\right\rangle \\
& =\frac{\hbar}{2} \int_{0}^{\infty} d \omega \hbar \omega \chi^{\prime \prime}(\omega) \\
& \times\left[r_{R} I_{L}(\omega) n_{L}(\hbar \omega)-r_{L} I_{R}(\omega) n_{R}(\hbar \omega)\right] .
\end{aligned}
$$

Note that if $\tilde{I}_{L}(\omega)=\tilde{I}_{R}(\omega)=\tilde{I}(\omega), r_{\nu}$ is simplified as $r_{\nu}=\alpha_{\nu} /\left(\alpha_{L}+\alpha_{R}\right)$. Hence, the above formula is reduced to $(5)$ in the main text.

Weak coupling limit $(\alpha \rightarrow 0)$. - Let $H_{s}$ be the system's Hamiltonian, i.e., $H_{s}=\hbar \Delta \sigma_{x} / 2$. It is diagonalized as

$$
e^{i \pi \sigma_{y} / 4} H_{s} e^{-i \pi \sigma_{y} / 4}=\hbar \Delta \sigma_{z} / 2 .
$$

We first express Segal and Nitzan's result [3], which is obtained from the quantum master equation:

$$
\langle J\rangle=\frac{\hbar \pi}{2} \frac{\Delta I_{L}(\Delta) I_{R}(\Delta)\left[n_{L}(\Delta)-n_{R}(\Delta)\right]}{I_{L}(\Delta)\left(2 n_{L}(\Delta)+1\right)+I_{R}(\Delta)\left(2 n_{R}(\Delta)+1\right)} .
$$

Note that the above expression is the first-order expression with respect to the coupling strength between the system and reservoirs. Then, we find the zeroth-order expression for $\chi^{\prime \prime}$, which implies the response function at the weak coupling limit. For the case of thermal conductance, we use the equilibrium value of $\chi^{\prime \prime}$ :

$$
\chi^{\prime \prime}(\omega) \rightarrow \pi /[\hbar(2 n(\omega)+1)] \delta(\omega-\Delta) .
$$

We note that contribution arises only from $\omega=\Delta$, which immediately implies $r_{\nu}=I_{\nu}(\Delta) /\left(I_{L}(\Delta)+I_{R}(\Delta)\right)$.
To obtain the expression at far-from-equilibrium (19), we replace the temperature in Eq.(20) with an effective temperature at the weak coupling limit between the reservoirs:

$$
\chi^{\prime \prime}(\omega) \rightarrow \pi /\left[\hbar\left(2 n_{\mathrm{eff}}(\omega)+1\right)\right] \delta(\omega-\Delta) .
$$

In order to obtain the effective temperature, we use the quantum master equation. We write the master equation in the representation diagonalizing the spin Hamiltonian:

$$
\begin{aligned}
\dot{\rho} & =i\left[\rho, \Delta \sigma_{z} / 2\right]-\sum_{\nu=L, R}\left\{\left[X, R_{\nu} \rho\right]+\left[X, R_{\nu} \rho\right]^{\dagger}\right\} \\
X & =-\sigma_{x}
\end{aligned}
$$

where the matrices $R$ are given by the $2 \times 2$ matrix:

$$
R_{\nu}=-I_{\nu}(\Delta)\left(\begin{array}{cc}
0, & n_{\nu}(\Delta) \\
-n_{\nu}(-\Delta), & 0
\end{array}\right)
$$

In case of a single reservoir, the equilibration is guaranteed by the detailed balance for the matrix $R_{\nu}$ :

$$
R_{\nu 1,2} / R_{\nu 2,1}=n_{\nu}(\Delta) /\left[-n_{\nu}(-\Delta)\right]=e^{-\beta_{\nu} \hbar \Delta} .
$$

Then, by analyzing the effective detailed balance for the nonequilibrium situation with two reservoirs, the effective temperature is obtained as

$$
e^{-\beta_{\mathrm{eff}} \hbar \Delta}=\frac{I_{L}(\Delta) n_{L}(\Delta)+I_{R}(\Delta) n_{R}(\Delta)}{-I_{L}(\Delta) n_{L}(-\Delta)-I_{R}(\Delta) n_{R}(-\Delta)} .
$$

From this, a simple manipulation yields

$$
\begin{aligned}
\chi^{\prime \prime}(\omega) & =\frac{I_{L}(\Delta)+I_{R}(\Delta)}{I_{L}(\Delta)\left[2 n_{L}(\Delta)+1\right]+I_{R}(\Delta)\left[2 n_{R}(\Delta)+1\right]} \\
& \times \pi / \hbar \delta(\omega-\Delta) .
\end{aligned}
$$

Combining the ratio $r_{\nu}=I_{\nu}(\Delta) /\left(I_{L}(\Delta)+I_{R}(\Delta)\right)$, one gets the result (19).

Ising model with long-range interaction. - The spinboson model can be mapped onto an Ising model with long-range exchange interaction. We start with the derivation of the partition function for the spin-boson model. For simplicity, we set $\hbar=1$. We first divide the Hamiltonian into two parts and create the following definition:

$$
\begin{aligned}
H= & \Delta \sigma_{x} / 2+H_{z} \\
H_{z}= & \frac{\sigma_{z}}{2} \sum_{\nu=L, R} \sum_{k} \lambda_{\nu k}\left(b_{\nu k}+b_{\nu k}^{\dagger}\right) \\
& +\sum_{\nu=L, R} \sum_{k} \omega_{\nu k} b_{\nu k}^{\dagger} b_{\nu k}, \\
\tilde{\sigma}_{x}(u):= & e^{i H_{z} u} \sigma_{x} e^{-i H_{z} u} .
\end{aligned}
$$

Then, the partition function $Z=\operatorname{Tr} e^{-\beta H}$ is divided into two parts: $Z=Z_{+}+Z_{-}$as

$$
\begin{aligned}
Z & =Z_{+}+Z_{-} \\
Z_{ \pm} & =\left\langle \pm\left|\operatorname{Tr}_{\text {boson }} e^{-\beta H}\right| \pm\right\rangle,
\end{aligned}
$$




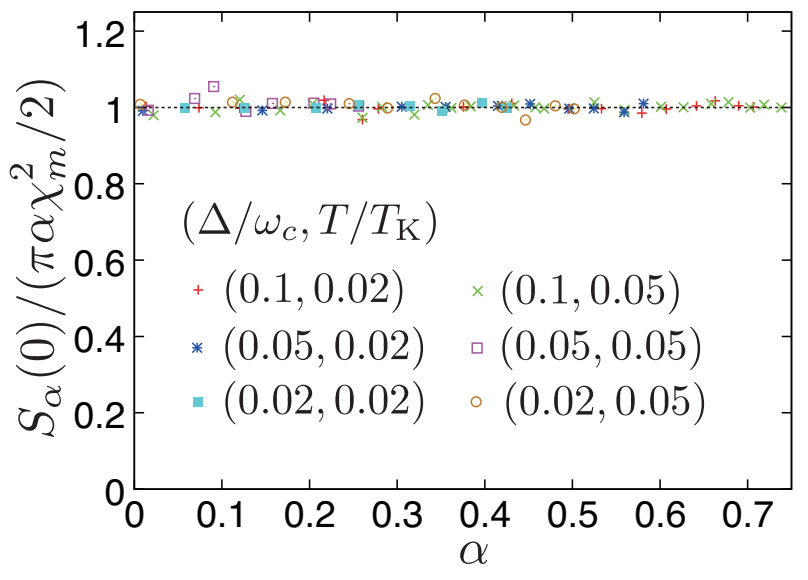

FIG. 5: (color online) Numerical validation of the Shiba relation.

where $| \pm\rangle$ is the eigenstate of $\sigma_{z}$, i.e., $\sigma_{z}| \pm\rangle= \pm 1| \pm\rangle$, and $\operatorname{Tr}_{\text {boson }}$ implies the trace with respect to boson's degrees of freedom. We expand $Z_{+}$as

$$
\begin{aligned}
Z_{+} & =\operatorname{Tr}_{\text {boson }}\left\{\left\langle+\left|e^{-\beta H_{z}} e_{\leftarrow}^{-\int_{0}^{\beta} d u \Delta \tilde{\sigma_{x}}(u) / 2}\right|+\right\rangle\right\} \\
& =\sum_{n=0}^{\infty} \operatorname{Tr}_{\text {boson }}\left\{\langle+| e^{-\beta H_{z}} \int_{0}^{\beta} d \tau_{1} \cdots \int_{0}^{\tau_{2 n-1}-\tau_{c}} d \tau_{2 n}\right. \\
& \left.\times\left(\frac{\Delta}{2}\right)^{2 n} \tilde{\sigma}_{x}\left(\tau_{1}\right) \cdots \tilde{\sigma}_{x}\left(\tau_{2 n}\right)|+\rangle\right\}
\end{aligned}
$$

where $\tau_{c}=1 / \omega_{c}$. By taking trace with respect to the boson part, we obtain a formal expression for $Z_{+}$[4, [5]:

$$
\begin{aligned}
Z_{+} & =Z_{0} \sum_{n=0}^{\infty}\left(\frac{\Delta \tau_{c}}{2}\right)^{2 n} \int_{0}^{\beta} \frac{d \tau_{1}}{\tau_{c}} \cdots \int_{0}^{\tau_{2 n-1}-\tau_{c}} \frac{d \tau_{2 n}}{\tau_{c}} \\
& \times \exp \left\{2 \alpha \sum_{i<j}(-1)^{i+j} \ln \left|\frac{\beta}{\pi \tau_{c}} \sin \left(\pi\left(\tau_{j}-\tau_{i}\right) / \beta\right)\right|\right\},
\end{aligned}
$$

where $Z_{0}$ is a partition function for the free boson part, and $Z_{-}$takes the same form. Finally, we connect this to the kink dynamics in the Ising model [5, 6]. The equivalent Ising dynamics is obtained when the imaginary time is considered to be a position of spin. By discretizing the space, the equivalent Ising Hamiltonian $H_{I}$ reads

$$
\beta_{I} H_{I}=-\frac{J_{n n}}{2} \sum_{i=1}^{N} \sigma_{i} \sigma_{i+1}-\frac{\alpha}{2} \sum_{j<i} \frac{(\pi / N)^{2} \sigma_{i} \sigma_{j}}{\sin ^{2}[\pi(j-i) / N]}
$$

where $\beta_{I}$ is the inverse temperature in the mapped Ising model. The nearest neighbor interaction coefficient $J_{n n}$ is given by $J_{n n}=-\alpha(1+\gamma)-\ln \left(\Delta \tau_{c} / 2\right)$, where $\gamma$ is Euler's constant. The lattice number $N$ is determined by $N=\beta \omega_{c}$, and hence the Monte Carlo simulation is possible only for $\beta \omega_{c} \gg 1$.
The Shiba relation and the cotunneling formula. - The Shiba relation [7], which is a general identity equation valid at low temperatures well below the Kondo temperature, is written by

$$
S_{\alpha}(0)=\frac{\alpha \pi \chi_{m}^{2}}{2}
$$

where $\chi_{m}$ is the susceptibility of the local spin. For the numerical validation, we calculate $S_{\alpha}(0)$ and $\chi_{m}$ at temperatures much lower than $T_{K}$, and show the ratio $S_{\alpha}(0) /\left(\alpha \pi \chi_{m}^{2} / 2\right)$ as a function of $\alpha$ for several sets of $(\Delta, T)$ in Fig. 5. The figure clearly demonstrates the validity of the Shiba relation, implying one of the evidences on the reliability of our numerical calculations.

By substituting the Shiba relation (35) into the thermal conductance (Eq. (6) in the main text), we obtain an approximation which is valid at low temperatures $\left(T \ll T_{K}\right)$

$$
\kappa \sim \frac{\pi k_{B} \hbar^{2} \chi_{m}^{2}}{8} \int_{0}^{\omega_{c}} d \omega I_{L}(\omega) I_{R}(\omega)\left[\frac{\beta \omega / 2}{\sinh (\beta \omega / 2)}\right]^{2}
$$

For weak coupling $\left(\alpha_{L}, \alpha_{R} \ll 1\right)$, the susceptibility $\chi_{m}$ is approximately given by $2 / \hbar \Delta$, which is the one for the isolated two-state system, and one can recover the cotunneling formula derived in Ref. [8]. For arbitrary coupling, however, the cotunneling formula of Ref. [8] becomes wrong, and needs consideration of strong renormalization by the Kondo effect. Actually, since the energy scale is renormalized from $\hbar \Delta$ to $k_{B} T_{K}$, one should take $\chi_{m} \sim 2 /\left(k_{B} T_{K}\right)$ to obtain the correct result of thermal conductance at low temperatures $\left(T \ll T_{K}\right)$.

Finally, we point out that the same cotunneling formula can be derived even when the local system is a harmonic oscillator by replacing $\Delta$ with the frequency of the local oscillator. This indicates that the low-temperature heat transport of the present model is governed by the fixed-point Hamiltonian of a local "harmonic oscillator" after nontrivial renormalization by the Kondo physics.

[1] F. Guinea, V. Hakim and A. Muramatsu, Phys. Rev. B 32, 4410 (1985); F. Guinea, Phys. Rev. B 32, 4486 (1985).

[2] T. A. Costi, Phys. Rev. Lett. 80, 1038 (1998).

[3] D. Segal and A. Nitzan, Phys. Rev. Lett. 94, 034301 (2005); J. Chem. Phys. 122, 194704 (2005).

[4] A. J. Leggett, S. Chakravarty, A. T. Dorsey, M. P. A. Fisher, A. Garg and W. Zwerger, Rev. Mod. Phys. 59, 1 (1987).

[5] K. Völker, Phys. Rev. B 58, 1862 (1998).

[6] J. Cardy, J. Phys. A 14, 1407 (1981).

[7] H. Shiba, Prog. Theor. Phys. 54, 967 (1975).

[8] T. Ruokola and T. Ojanen, Phys. Rev. B 83, 045417 (2011). 